\title{
Universality and Breaking of Universality in Photo-induced Non-equilibrium Phase Transitions
}

Chong-Yu Ruan ${ }^{1}$, Shuaishuai Sun ${ }^{1}$, Joseph Williams ${ }^{1}$, Faran Zhou ${ }^{1}$, Ming Zhang ${ }^{1}$, Xiaoyi Sun ${ }^{1}$, Christos Malliakas $^{2}$, Mercouri Kanatzidis ${ }^{2}$ and Mohammad Maghrebi ${ }^{1}$

${ }^{1}$ Michigan State University, East Lansing, Michigan, United States, ${ }^{2}$ Northwestern University, Evanston, Illinois, United States

Nonequilibrium phase transition plays a pivotal role in a broad physical context from condensed matter to cosmology. On a smaller scale, the quantum simulator experiments afforded by ultracold atoms have started a new era of non-equilibrium systems[1]. A generic non-equilibrium setting is one where a system parameter (such as the interaction strength) suddenly changes, leading to the evolution of the many-body state of the system. Here, we demonstrate, for the first time, a charge-density-wave (CDW) state in 1T$\mathrm{TaS}_{2}$ [2] that, upon a sudden interaction quench, exhibits a universal, critical behavior far from equilibrium. The quench in our experiment is engineered through ultrafast infrared laser pulses, which drive the initial near-commensurate (NC) state towards a thermal (incommensurate) state. We unambiguously show that the system, after a fast evolution at short times, exhibits a universal behavior in two distinct stages: prethermalization characterized by a long plateau where the order parameters do no evolve followed by thermalization where the new state undergoes coarsening. We capture the entire nonequilibrium self-organization dynamics through highly sensitive, femtosecond coherent electron scattering experiments $[3,4]$. The most surprising feature of our experiment is the emergence of a new dynamical pathway, through a nonthermal critical state, that effectively changes the diffusion constant characteristic of the thermalization dynamics - by more than an order of magnitude. In another instance, we can suppress such universal behaviors by changing the excitation wave-length to mid-infrared. The lack of universality in this case is mediated by suppressing the excessive thermal fluctuations in the system to boost the cooperativity, yielding an even more efficient pathway in forging the new broken-symmetry ground state out of equilibrium. Our quantitative measurements not only provide a benchmark for studying non-equilibrium phase transitions in condensed matter systems, but also opens an intriguing perspective of controlling quantum materials on ultrashort timescales that are useful for practical applications.

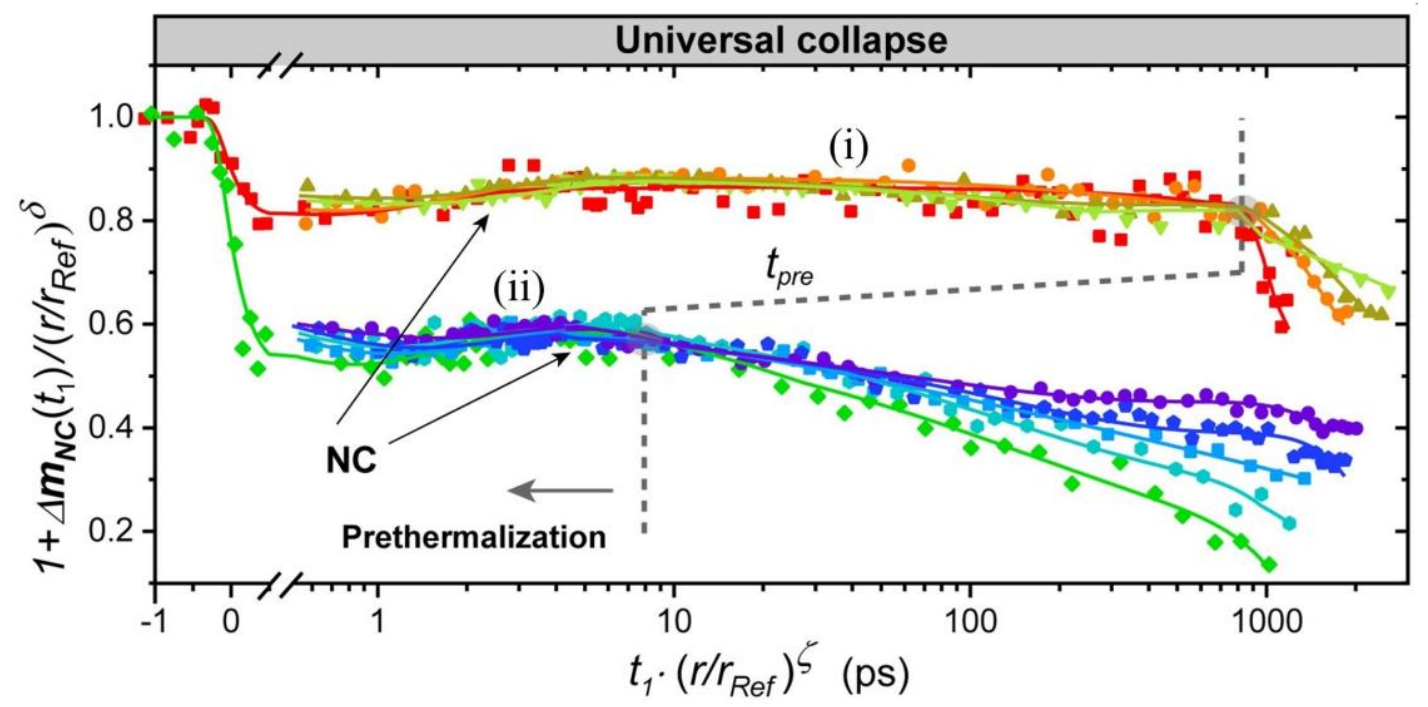


Figure 1. Universal dynamics of the CDW order parameter. The universal collapse of the order parameter evolution in the prethermalization window in two different scaling regimes: (i) $\mathrm{Fc}, 1<\mathrm{F}<\mathrm{Fc}, 2$ and (ii) $\mathrm{Fc}, 2<\mathrm{F}$, based on dynamical and amplitude exponents $\zeta$ and $\delta$. For the dynamical scaling, we define $\mathrm{t} 1=\mathrm{t}-$ $\mathrm{ti}$, where $\mathrm{ti}=500 \mathrm{fs}$ is subtracted to exclude the initial overdamped dynamics.

References

[1] Gring, M. et al. Science 337, 1318 (2012).

[2] T.R. T. Han et al., Sci. Adv. 1, e1400173 (2015).

[3] J. Williams et al., Struc. Dyn. 4, 044035 (2017).

[4] F. Zhou et al., arXiv 1904.07120 (2019). 